\title{
Reducing the Foreign Body Reaction by Surface Modification with Collagen/Hyaluronic Acid Multilayered Films
}

\author{
Cindy Yi Chi Hsieh, ${ }^{1}$ Fang-Wei Hu, ${ }^{1}$ Wen-Shiang Chen, ${ }^{2}$ and Wei-Bor Tsai ${ }^{1}$ \\ ${ }^{1}$ Department of Chemical Engineering, National Taiwan University, Taipei 106, Taiwan \\ ${ }^{2}$ Department of Physical Medicine and Rehabilitation of National Taiwan University Hospital, Taipei 100, Taiwan \\ Correspondence should be addressed to Wei-Bor Tsai; weibortsai@ntu.edu.tw
}

Received 3 December 2013; Accepted 6 January 2014; Published 13 April 2014

Academic Editors: S. Lamponi, S. Liao, and J. Wang

Copyright (c) 2014 Cindy Yi Chi Hsieh et al. This is an open access article distributed under the Creative Commons Attribution License, which permits unrestricted use, distribution, and reproduction in any medium, provided the original work is properly cited.

Biological response against foreign implants often leads to encapsulation, possibly resulting in malfunction of implants devices. The aim of this study was to reduce the foreign body reaction by surface modification of biomaterials through layer-by-layer deposition of type I collagen (COL)/hyaluronic acid (HA) multilayer films. Polydimethylsiloxane (PDMS) samples were coated with alternative COL and HA layers with different layers. We found that the in vitro adhesion, proliferation, and activation of macrophage-like cells were greatly decreased by COL/HA multilayered deposition. The PDMS samples modified with 20 bilayers of COL/HA were implanted in rats for 3 weeks, and the thickness of encapsulation surrounding the samples was decreased by $29-57 \%$ compared to the control unmodified PDMS. This study demonstrates the potential of COL/HA multilayer films to reduce foreign body reaction.

\section{Introduction}

Biological response against artificial implants leads to encapsulation of implants, which often causes malfunction of implants or patients' agonies due to capsular contracture [1-3]. For example, capsule contracture of fibrous tissues overlying silicone breast implants may cause the implant rupture and become painful [3]. When a foreign material is implanted into a host, the interaction between the material and the surrounding injured tissues causes acute inflammation, similar to the natural healing process that usually lasts for a week $[1,2]$. Chronic inflammation with the participation of monocytes, macrophages, and foreign body giant cells (FBGCs) may occur with a duration of less than 3 weeks if the wound is not healed [1]. Macrophages try to eliminate the foreign object from the host. However, the implant is too large to be phagocytosized, so activated macrophages fuse together to form multinucleated FBGCs [1]. The inability of FGBCs in eliminating the foreign object will lead to a route to encapsulation of the implant with dense collagen matrix that is secreted by fibroblasts in order to wall the foreign object off the host $[1,2]$.

Macrophages can be considered as a hallmark due to their critical roles in guiding through the entire wound healing process $[2,4]$. Activated macrophages release biosignals such as interleukins to induce the formation of FBGCs as well as collagen synthesis from fibroblasts. To prevent these undesirable events from occurring, scientists have sought suitable surface modifications on biomaterials to prevent capsule formation by altering the hydrophilicity, topology, roughness, and surface chemistry of surfaces $[5,6]$. Strategies in inhibiting nonspecific protein adsorption and recruitment of neutrophil and macrophage to biomaterials are attractive since proteins and leucocytes play important roles in the foreign body reaction, which eventually results in fibrosis. Thus, it is suggested that reduction of macrophage adhesion and activation may reduce the formation of the foreign body reaction and capsule formation $[1,7,8]$.

Natural polymers are usually thought to be biocompatible and suitable for biomedical applications. Among natural polymers, collagen (COL) and hyaluronic acid (HA), the main components in the extracellular matrix, are frequently used to prepare biomimicking coatings to improve the biocompatibility of biomedical devices [9]. Previous studies have shown the potential of high-molecular-weight HA in limiting the extent of injury in lung epithelial cells, promoting repair of parenchymal cell damage, and inhibiting apoptosis $[10,11]$. It is shown that macrophages disappeared in an injured site 
with direct injection of HA, while macrophages reappeared with the depletion of HA [12]. Similarly, type I COL was found to inhibit the proliferation of RAW 264.7 cells and to lessen the degree of the foreign body reaction $[3,13,14]$. Therefore, we suggest that surface modification of implants with COL and HA may diminish the foreign body reaction.

The aim of the current study was to evaluate the modification of COL/HA on a polydimethylsiloxane (PDMS), a silicone that is widely used in breast implants, for reducing the foreign body reaction. PDMS substrates were modified with COL and HA by a simple layer-by-layer (LBL) deposition. This technique is based on alternate adsorption of positively and negatively charged macromolecules to build an ultrathin polyelectrolyte multilayer (PEM) film onto a substratum [15]. The water-based approach is biocompatible and is not restricted to types, sizes, and shapes of substrates. Many studies indicated that cell behavior can be controlled by the chemical and physical properties of PEM films [16-20], which can be tuned by adjusting deposition parameters such as nature of polyelectrolytes, $\mathrm{pH}$, and ionic strength of polyelectrolyte solutions [21]. Positively charged COL and negatively charged HA can be deposited onto PDMS via this simple process with precise control in thickness and surface charge [22]. This study is the first investigation on the efficacy of COL/HA films for reducing the foreign body reaction. In vitro adhesion and activation of macrophages and formation of collagen capsule in a rat model were evaluated on COL/HA deposited PDMS.

\section{Materials and Methods}

2.1. Materials and Buffers. Reagents were received from Sigma-Aldrich (St. Louis, USA) unless specified otherwise. Ethidium homodimer-1 (EthD-1) was purchased from Fluka, USA. N-(1-Naphthyl)-ethylenediamine dihydrochloride (NED) was purchased from R.D.H. (Germany). Porcine collagen $(3.0 \mathrm{mg} / \mathrm{mL}, \mathrm{pH} \sim 2.3)$, obtained from SunMax biotechnology (Tainan, Taiwan), was purified from specific pathogen free porcine skin and contained 96-98\% type I collagen with the remainder being type III collagen. Medical grade hyaluronic acid (Mw 1,500 kDa) was received from Maxigen Biotechnology Inc. (Taiwan). Polydimethylsiloxane (PDMS, Sylgard 184) was purchased from Dow Corning (USA).

Cell culture medium consisted of high glucose $\alpha$-MEM (HyClone, USA), 10\% (v/v) fetal bovine serum (JRH, Australia), $25 \mu \mathrm{g} / \mathrm{mL}$ amphotericin B (fungizone, GIBCO, USA), $200 \mu \mathrm{g} / \mathrm{mL}$ gentamycin (GIBCO), and $1 \mathrm{mM}$ sodium pyruvate. Trypsin-EDTA solution was obtained from GIBCO. The lactate dehydrogenase $(\mathrm{LDH})$ solution contained $3.6 \%$ sodium lactate, $0.3 \% \mathrm{NAD}^{+}, 0.27 \%$ diaphorase, $0.03 \%$ bovine serum albumin, $1.2 \%$ sucrose, and $0.02 \%$ indonitrotetrazolium. Scott solution was prepared by dissolving $2 \mathrm{~g}$ $\mathrm{NaHCO}_{3}$ and $20 \mathrm{~g} \mathrm{MgSO}_{4} \cdot 7 \mathrm{H}_{2} \mathrm{O}$ in $1000 \mathrm{~mL}$ deionized water. Live and dead reagent was prepared by mixing $10 \mu \mathrm{L}$ calcein AM solution ( $1 \mathrm{mg}$ calcein AM in $503 \mathrm{~mL}$ DMSO), $20 \mu \mathrm{L}$ EthD-1 solution (1 mg EthD-1 in $582 \mathrm{~mL}$ DMSO), and $10 \mu \mathrm{L}$ of $\mathrm{Ca}^{2+}$ and $\mathrm{Mg}^{2+}$ supplemented phosphate buffered saline (PBS; $0.05 \mathrm{~g} \mathrm{CaCl}_{2}$ and $0.05 \mathrm{~g} \mathrm{MgCl}_{2} \cdot 6 \mathrm{H}_{2} \mathrm{O}$ in $500 \mathrm{~mL}$ PBS at $\mathrm{pH} 7.4)$.
2.2. Preparation of PDMS Samples. Part A/Part B of Sylgard 184 was mixed at ratio of $10: 1$ to prepare PDMS elastomers according to the manufacturer's instruction. To prepare samples for in vitro cell study, PDMS was formed in 24-wells TCPS plate by adding $150 \mu \mathrm{L}$ of Sylgard 184 mixture into each well and then cured at $60^{\circ} \mathrm{C}$ overnight. For in vivo implantation study, $4.2 \mathrm{~g}$ of Sylgard 184 mixture was poured into an aluminum dish $(5.1 \mathrm{~cm}$ in diameter) and then cured in a $60^{\circ} \mathrm{C}$ oven. The PDMS elastomer was shaped into 5-mm disks with $2 \mathrm{~mm}$ in thickness. The PDMS samples were cleaned in Liqunox (Valconox, USA) and stored in deionized water.

\subsection{Preparation and Characterization of COL/HA Multi-} layer Films. PDMS substrates were treated with radio frequency glow discharge oxygen plasma $(50 \mathrm{~W}, 70$ mtorr, $2 \mathrm{~min}$, $23.6 \mathrm{sccm}$ ) in order to enhance their hydrophilicity [23]. The plasma-treated PDMS samples were then immersed in deionized water until COL/HA deposition. COL and HA solutions were prepared as $0.5 \mathrm{mg} / \mathrm{mL}$ in $0.1 \mathrm{M}$ acetate buffer ( $\mathrm{pH} 4.0$ ) and filtered through $0.22 \mu \mathrm{m}$ filters. The substrates were first immersed in COL solution for $10 \mathrm{~min}$, followed by two rinses with $0.1 \mathrm{M}$ acetate buffer ( $\mathrm{pH} 4.0$ ). Next, the PDMS substrates were immersed in HA solution for $10 \mathrm{~min}$, followed by the same rinsing procedure. The deposition/rinsing cycles were repeated until the desired numbers of COL/HA bilayers were finished. All COL/HA multilayered films used in this study proceeded with HA as the topmost layer. The COL/HAcoated PDMS were air-dried and stored at room temperature until usage. Prior to in vitro cell culture or in vivo implantation, the samples were sterilized in $70 \%$ ethanol and rinsed with PBS. The multilayer films of COL and HA were represented by $[\mathrm{COL} / \mathrm{HA}]_{n}$, where $n$ represents bilayer numbers.

The deposition of COL/HA multilayer films was analyzed by quartz crystal microbalance (QCM). The QCM apparatus and quartz crystals with a resonance frequency of ca. $9 \mathrm{MHz}$ were purchased from ANT Inc. (Taipei, Taiwan). The gold electrodes for the QCM head were first immersed in $10 \mathrm{mM} 3-$ mercapto-1-propansulfonic acid solution in deionized water at room temperature for 1 day in order to create a negatively charged sulfonated substrate to facilitate the deposition of a layer of polyethylene imine (PEI, MW 750,000). Then, the functionalized substrates were alternately immersed in $\mathrm{HA}$ and COL solutions each for $10 \mathrm{~min}$ until the desired bilayer numbers were reached. After being proceeded with sterilization in 70\% ethanol and air-dried, the QCM heads deposited with COL/HA multilayer films were inserted into the QCM apparatus for resonance frequency measurement. The resonance frequency was recorded after equilibrium at which the frequency shift rate was within $0.1 \mathrm{~Hz} / \mathrm{min}$. The adsorbed mass was calculated from frequency shift according to the Sauerbrey equation [18].

2.4. The Adhesion and Activation of RAW 264.7 Cells. Raw 264.7 murine monocyte/macrophage cell line was obtained from Food Industry Research and Development Institute (Hsin Chu, Taiwan) and cultured under $37^{\circ} \mathrm{C}$ in $5 \% \mathrm{CO}_{2}$ atmosphere by standard sterile cell culture procedures. 
The cell seeding densities were $6 \times 10^{4}$ cells $/ \mathrm{cm}^{2}$ for cell adhesion/cytotoxicity analysis and $3 \times 10^{5}$ cells $/ \mathrm{cm}^{2}$ for nitric oxide (NO) generation experiment.

Cell adhesion was determined by an LDH assay, modified from a previous procedure [24]. Cells cultured on different substrates for 24 or $48 \mathrm{~h}$ were lysed with $0.1 \%$ triton-X100 for $20 \mathrm{~min}$. $50 \mu \mathrm{L}$ supernatant of the cell lysates after centrifugation at $1000 \mathrm{rpm}$ was mixed with $60 \mu \mathrm{L} \mathrm{LDH}$ solution. The reaction was undergone at $37^{\circ} \mathrm{C}$ for $5 \mathrm{~min}$ in the dark and then stopped by adding $20 \mu \mathrm{L}$ of $1.6 \%$ sodium oxamate. The absorbance at $490 \mathrm{~nm}$ was measured by an ELISA reader (Bio-Tek, EL800, USA). A standard curve was generated with a series of cell solutions with known cell densities.

Cytotoxicity of COL/HA multilayer films was evaluated by staining of cell culture with calcein AM (for living cells) and EthD-1 (for dead cells). The cells were cultured on different substrates for $24 \mathrm{~h}$, followed by incubation with the fluorescent dye for $30 \mathrm{~min}$ at $37^{\circ} \mathrm{C}$. The reagent was then rinsed with PBS and cellular uptake of the fluorescent dyes was analyzed by fluorescent microscopy. The excitation and emission wavelengths for calcein AM were $488 \mathrm{~nm}$ and 505$530 \mathrm{~nm}$, respectively, while those for EthD-1 were $543 \mathrm{~nm}$ and $560 \mathrm{~nm}$, respectively.

The extent of activation in RAW 265.7 cells adhering on the substrates was evaluated by nitric oxide (NO) release. First, cells were seeded and incubated on the substrates for $12 \mathrm{~h}$. Then the culture medium supplemented with lipopolysaccharide (LPS) replaced the culture medium for another $24 \mathrm{~h}$ of culture. Next, the cell medium was transferred into 96-wells plate and analyzed using Griess reagent (Promega, USA). In brief, equal volume of $1 \%$ sulfamide in $5 \%$ phosphoric acid in deionized water was added to the cell medium, followed by an addition of $0.1 \%$ NED solution for reaction. NO release was analyzed by the absorbance of $570 \mathrm{~nm}$.

2.5. In Vivo Evaluation in a Rat Model. The animal procedure followed the ethical guidelines of Care and Use of Laboratory Animals (National Taiwan University, National Institutes of Health Publication number 85-23, revised 1985) and was approved by the Animal Center Committee of National Taiwan University. Prior to implantation, the samples were sterilized in $70 \%$ ethanol and then soaked in sterilized PBS. Male Wistar rats weighting $\sim 500 \mathrm{~g}$ were first anesthetized by intravenous injection of ketamine. The bilateral sides of the backs of rats were shaved, depilated, and disinfected with $70 \%$ iodine. Next, the samples were then subcutaneously inserted into incisions made on the bilateral sides, as shown in Figure 1(a). After 3 weeks, the rats were sacrificed and the implants along with their surrounding tissues were excised.

The specimens were processed by standard histological techniques. Briefly, the PDMS samples along the surrounding tissues were first dehydrated by soaking in a series of ethanol solutions (from $0 \%$ to $95 \%$; 30 min each) and $100 \%$ ethanol (overnight). The samples were then soaked in xylene before placing them in $60^{\circ} \mathrm{C}$ paraffin wax for $2 \mathrm{~h}$, followed by cooling in an ice bath. The samples were then sliced in half in order to remove the PDMS samples from the tissues and then

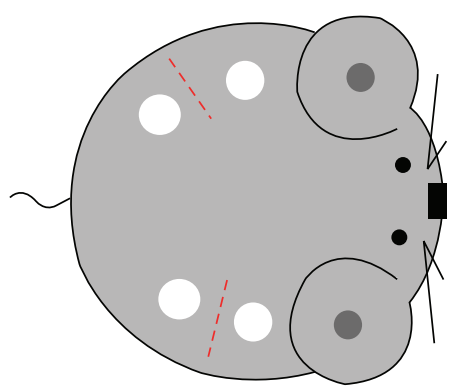

(a)

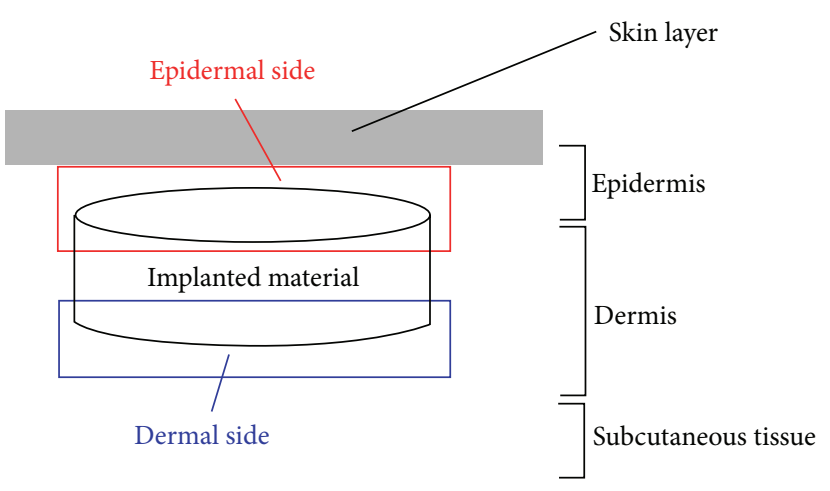

(b)

FIgURE 1: (a) A schematic sketch of the implanted sites in rats. The white circles represent the implanted samples. (b) A schematic sketch of the tissues around an implant, where the epidermis side towards strata cornea is denoted as the epidermal layer and the dermal side towards hypodermis is denoted as the dermal layer.

embedded in paraffin wax again. Tissue slides were prepared with microtome incision of embedded tissues into $6 \mu \mathrm{m}$ thick sections. The tissue slides were rehydrated through series of soaking in xylene, $100 \%$ to $30 \%$ ethanol solutions (10 min each), and finally deionized water (twice, $10 \mathrm{~min}$ ). The tissue slides were stained in Mayer's hematoxylin for $15 \mathrm{~min}$, followed by soaking in Scott solution for $3 \mathrm{~min}$, and rinsed with deionized water. Then, tissue slides were stained in Eosin solution for $30 \mathrm{~s}$, followed by a series of soaking in $95 \%, 100 \%$ ethanol and xylene, sequentially. Finally, the stained tissue slides were mounted for microscopic observation.

The slides of the surrounding tissue on two sides of each sample, the epidermal layer towards skin and the dermal layer towards hypodermis (Figure 1(b)), were examined under a microscope (200x in magnitude). The outline of dense capsules in each image was traced manually, and the areas of the dense capsules were determined by NIH Image J software. The thickness of a dense capsule was calculated by the ratio of the area to the length. Four images were selected randomly for each sample for measurement.

2.6. Statistical Analysis. All statistical data were performed with the GraphPad InStat software (Instat 3.0, GraphPad Software, USA) by Student-Newman-Keuls multiple comparisons test. $P \leqq 0.05$ was considered as a significant difference. 


\section{Results and Discussion}

3.1. Development of COL/HA Multilayered Films. QCM measurement was applied to investigate the development of COL/HA multilayered films in this study. However, the base substrate of QCM chips is gold, which is quite different from the PDMS substrate. Unfortunately, it is very difficult to duplicate the PDMS surface on the gold substrate. Therefore, the results from the QCM measurement are not necessary to represent the actual deposition of COL/HA multilayer films on the PDMS samples. Nevertheless, we think that the results could provide a clue for the growth of COL/HA multilayer films.

The development of COL/HA multilayer films showed an approximate linear growth on the adsorbed mass in respect of bilayer numbers between 45,86 , and $200 \mu \mathrm{g} / \mathrm{cm}^{2}$ for 5,10 , and 20 bilayers of COL/HA films, respectively (Figure 2). The trend of linear increment with respect to bilayer numbers is consistent with the results from previous studies $[9,22]$. Therefore, we expect that the deposition of COL/HA multilayer films on PDMS should be established in a similar manner.

3.2. The Adhesion and Activation of RAW 265.7 Cells on the COL/HA Multilayer Films. Compared to the PDMS control, COL/HA multilayered films decreased the adhesion of RAW265.7 cells (Figure 3). On day 1, the decrease in the cell numbers on the substrates with 5, 10, and 20 bilayers of COL/HA films was about $33 \%, 9 \%$, and $68 \%$, respectively, compared to the unmodified PDMS. As for 2-day culture, greater reduction in the cell numbers was observed on the substrates coated with COL/HA multilayered films. The maximal inhibition in cell adhesion appeared on $[\mathrm{COL} / \mathrm{HA}]_{20}$, while the efficacy of $[\mathrm{COL} / \mathrm{HA}]_{5}$ and $[\mathrm{COL} / \mathrm{HA}]_{10}$ in resisting cell adhesion was similar. The number of RAW265.7 cells did not increase during the second day of culture on $[\mathrm{COL} / \mathrm{HA}]_{20}$, while the cells on $[\mathrm{COL} / \mathrm{HA}]_{5}$ and $[\mathrm{COL} / \mathrm{HA}]_{10}$ proliferated at different extents.

Since the cease in cell proliferation on $[\mathrm{COL} / \mathrm{HA}]_{20}$ may be due to potential cytotoxicity of the films, the live and dead staining was used to verify the liability of the cells on $[\mathrm{COL} / \mathrm{HA}]_{20}$. We hardly found any dead cell on all COL/HA modified substrates, while more than $98 \%$ of the cells were found alive on the PDMS control. The result indicates that the decrease in cell numbers on the COL/HA deposited substrates is not due to cytotoxicity of the COL/HA multilayer coating.

The morphology of RAW265.7 cells on different substrates was observed using phase contrast microscopy (Figure 4). The cells on TPCS and the untreated PDMS were mostly observed to be round with slight adhesion and spreading morphology. Nevertheless, some RAW265.7 cells on the untreated PDMS displayed dendritic morphology, a sign of activation. On the other hand, the cells on the COL/HA modified substrates showed rounded morphology without any sign of adhesion and spreading. Furthermore, cell aggregates were found on the COL/HA modified substrates, with increment in aggregation as the COL/HA multilayer films thickened from 5, 10 to 20 bilayers. The results indicate that

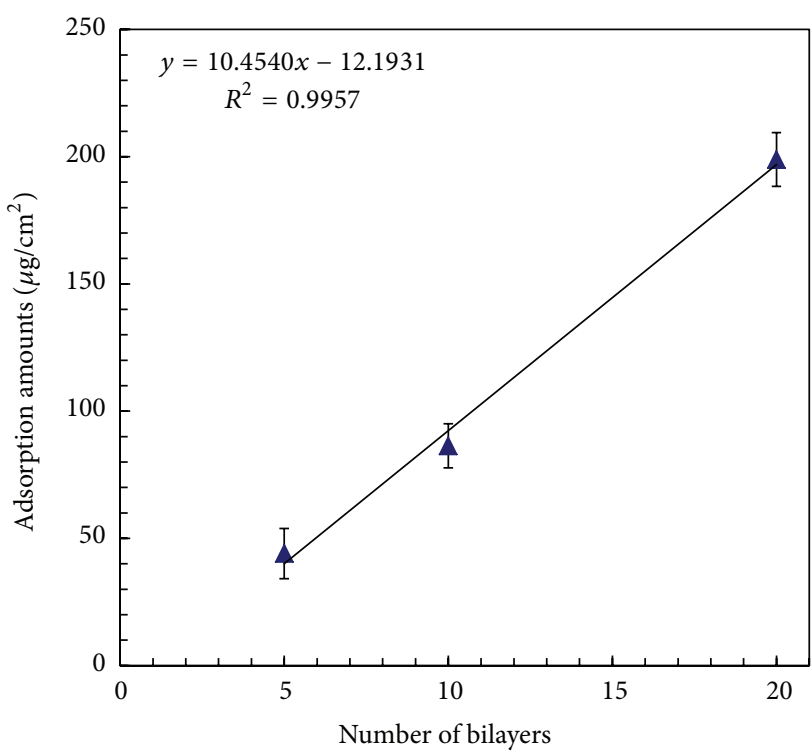

FIGURE 2: Mass accumulation of COL/HA multilayer films determined by QCM. The adsorbed mass was calculated from frequency shift according to the Sauerbrey equation.

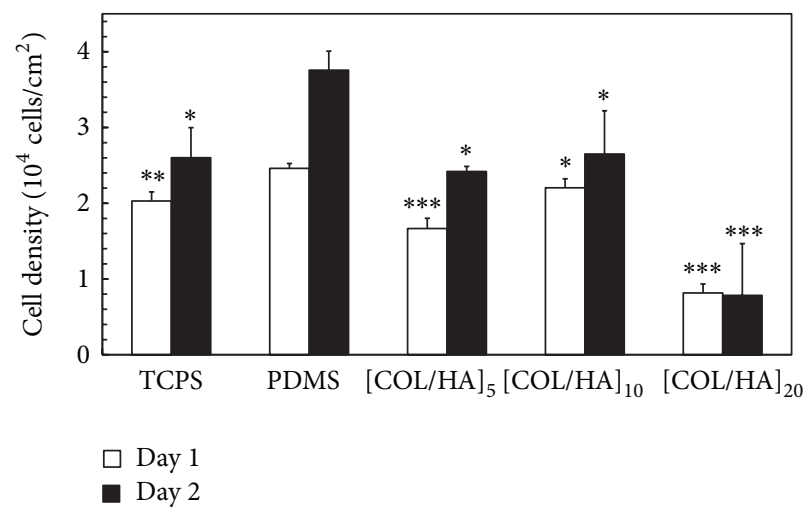

FIGURE 3: The adhesion of RAW 264.7 cells on the untreated and COL/HA coated PDMS after 1 or 2 days of culture. Multilayer films of COL/HA are represented by $[\mathrm{COL} / \mathrm{HA}]_{n}$, where $n$ represents the number of bilayer number from 5,10 to 20 . Cell seeding density was $2 \times 10^{4}$ cells $/ \mathrm{cm}^{2}$, and each sample was in triplicate. ${ }^{*},{ }^{* *}$, and ${ }^{* * *}$ represent $P<0.05,0.01$, and 0.001 , respectively, versus PDMS at the same culturing time.

the deposition of COL/HA multilayer films decreases the adhesion, proliferation, and activation of RAW265.7 cells compared to the untreated PDMS.

The decrease in cell affinity of COL/HA multilayered films with increasing bilayer numbers is consistent with the results on other polyelectrolyte multilayer (PEM) systems $[17,25,26]$. The bioinertness of the PEM films is correlated well with their thickness. For example, a dried PEM film on the order of $50 \mathrm{~nm}$ ( 20 layers) is effective to hinder cell spreading on the TCPS or gelatin [27]. As shown in this study, COL/HA films with 20 bilayers decrease cell adhesion more than the COL/HA films with fewer bilayers possess. Several explanations for such phenomenon include film hydration, 

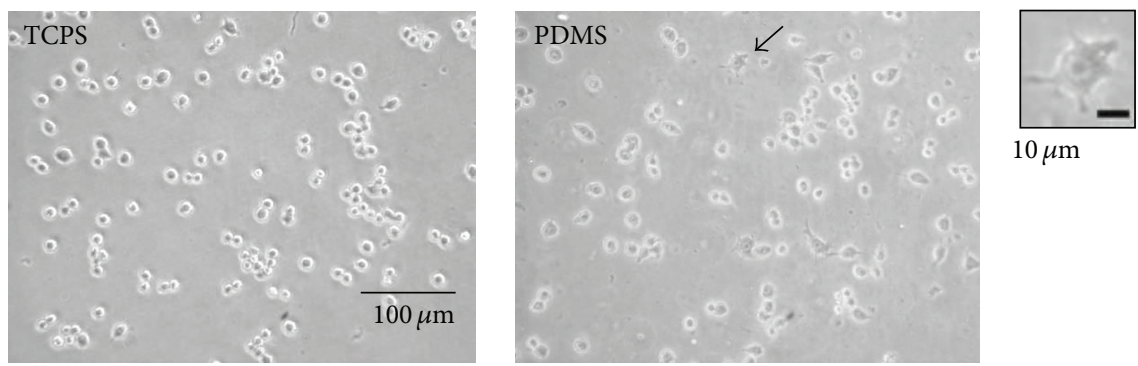

$10 \mu \mathrm{m}$
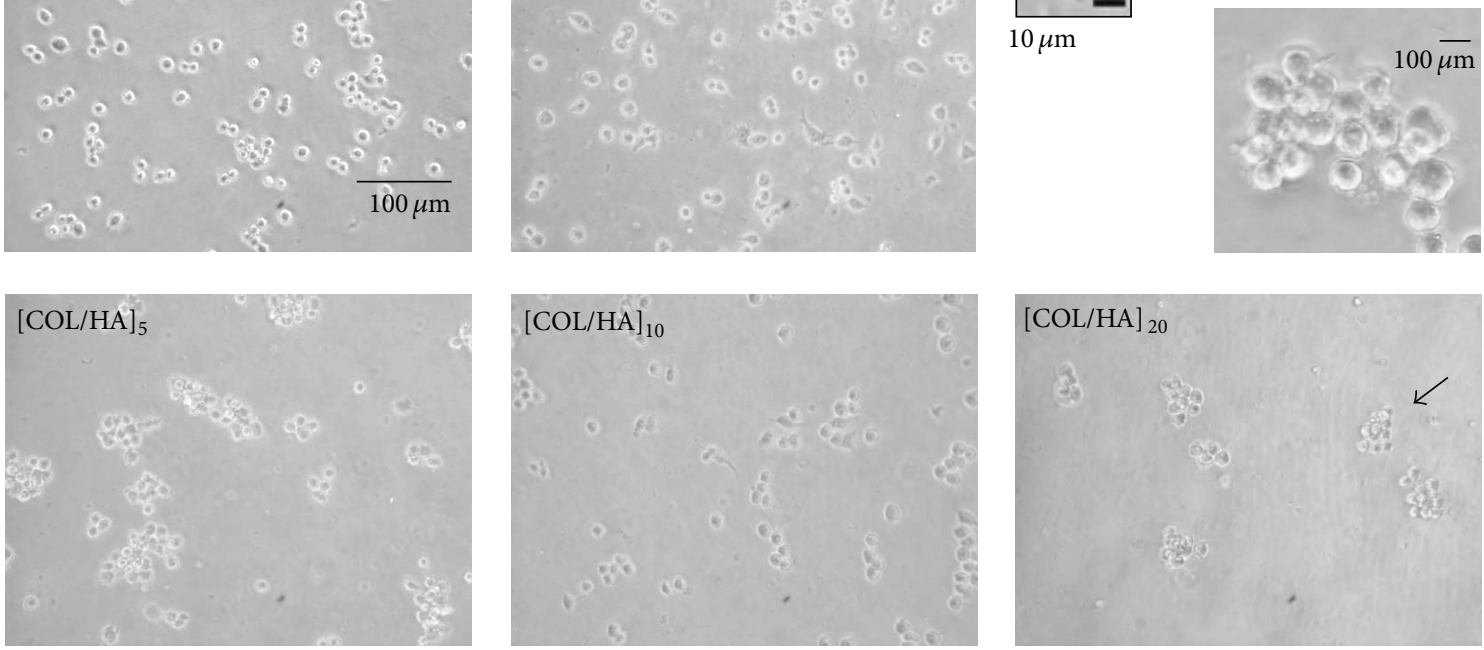

Figure 4: Microscopic images showed the morphology of RAW 264.7 cells on different substrates after 1 day of culture (200x for the large images and $400 \mathrm{x}$ for the inserted images). Arrows indicate the areas in the inserts. Multilayer films of COL/HA is represented by $[\mathrm{COL} / \mathrm{HA}]_{n}$, where $n$ represents the number of bilayer number from 5,10 to 20 .

swelling, and changes in the elastic modulus [28]. In general, cells form more stable focal adhesions on stiffer substrates [29]. The thicknesses of the PEM assembles is negatively correlated with their stiffness [30]. Besides the overall physical properties of PEM films, the types of polyelectrolytes chosen in this study may also contribute to their bioinertness towards macrophages. Type I collagen has been shown to completely block the progression of macrophage cell cycle through inhibition of the $G_{1} \rightarrow S$ phase transition [31]. HA has been shown to consist dual anti-inflammatory mechanism with a regulatory role in macrophage proliferation and death [32]. Therefore with the characteristics of type I collagen and hyaluronic acid, COL/HA multilayer films are unfavorable to the adhesion, proliferation, and activation of macrophages.

As shown previously, RAW265.7 cells adhering to the untreated PDMS showed a sign of activation with spreading and dendritic morphology, while the cells culturing on COL/HA multilayer films did not seem to be activated in terms of morphology. The production of NO by RAW265.7 cells was further evaluated on $[\mathrm{COL} / \mathrm{HA}]_{20}$. LPS was added to stimulate macrophage activation, otherwise the NO production was too low to differentiate the effect of $[\mathrm{COL} / \mathrm{HA}]_{20}$. The addition of LPS decreased cell numbers after $24 \mathrm{~h}$ of culture (Figure 5(a), $P<0.001$ versus NO LPS), which is consistent with previous reports that LPS increases cell apoptosis or inhibits cell proliferation [33]. RAW265.7 cells became more spreading with extrusions on PDMS in the presence of LPS (Figure 5(b)). On the other hand, most of the cells remained aggregated morphology on $[\mathrm{COL} / \mathrm{HA}]_{20}$, although some cells showed spread and activated morphology under LPS stimulation. NO production was stimulated by LPS addition and increased with the LPS dosage but was attenuated on $[\mathrm{COL} / \mathrm{HA}]_{20}$ (Figure 5(c)). Approximately $60 \%$ and $40 \%$ decrement in NO production were observed on $[\mathrm{COL} / \mathrm{HA}]_{20}$ in addition of 50 and $500 \mathrm{ng} / \mathrm{mL}$ of LPS, respectively, compared to the PDMS substrates with the addition of LPS $(P<0.001)$. The results suggest that COL/HA multilayered films possess a certain extent of antiinflammatory effect.

The anti-inflammatory effect might come from the physical and chemical properties of COL/HA multilayered films. The reduction in cell attachment to COL/HA multilayered films may affect the subsequent cellular events such as proliferation and differentiation [34, 35]. Furthermore, many researches have indicated that high-molecular-weight HA $\left(>10^{6} \mathrm{Da}\right)$ displays an anti-inflammatory effect, and HA reduces the production of activate oxygen species from macrophages [36]. However, type I COL is proinflammatory since it activates all three types of MAP kinases and upregulates the expression of the genes associated with inflammatory responses $[37,38]$. Nevertheless, the overall properties of COL/HA multilayer films effectively decrease the adhesion, proliferation, and activation of macrophages. Since NO participates in the immune response as a promoter for fibroblast migration, proliferation, and collagen deposition during wound healing $[39,40]$, the decrease in NO production on COL/HA multilayer films may reduce capsule formation in vivo. Therefore, we next evaluated the efficacy of $\mathrm{COL} / \mathrm{HA}$ films in reducing capsule formation in vivo.

3.3. In Vivo Evaluation in a Rat Model. The untreated PDMS and $[\mathrm{COL} / \mathrm{HA}]_{20}$-coated PDMS were implanted under bilateral sides of the backs of rats and explanted after 3 weeks of implantation. In the excised subcutaneous tissue surrounding implanted samples, it was observed that all samples were surrounded by fibrous capsules. The capsules identified as dense connective tissues that consist of fibrocytes, collagen, and blood vessels with some common inflammatory cells [41]. 


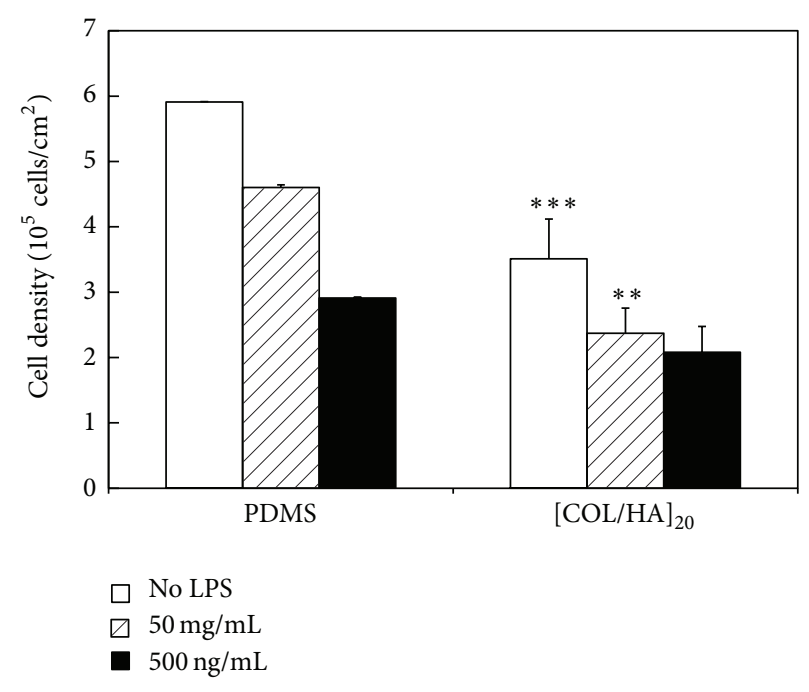

(a)
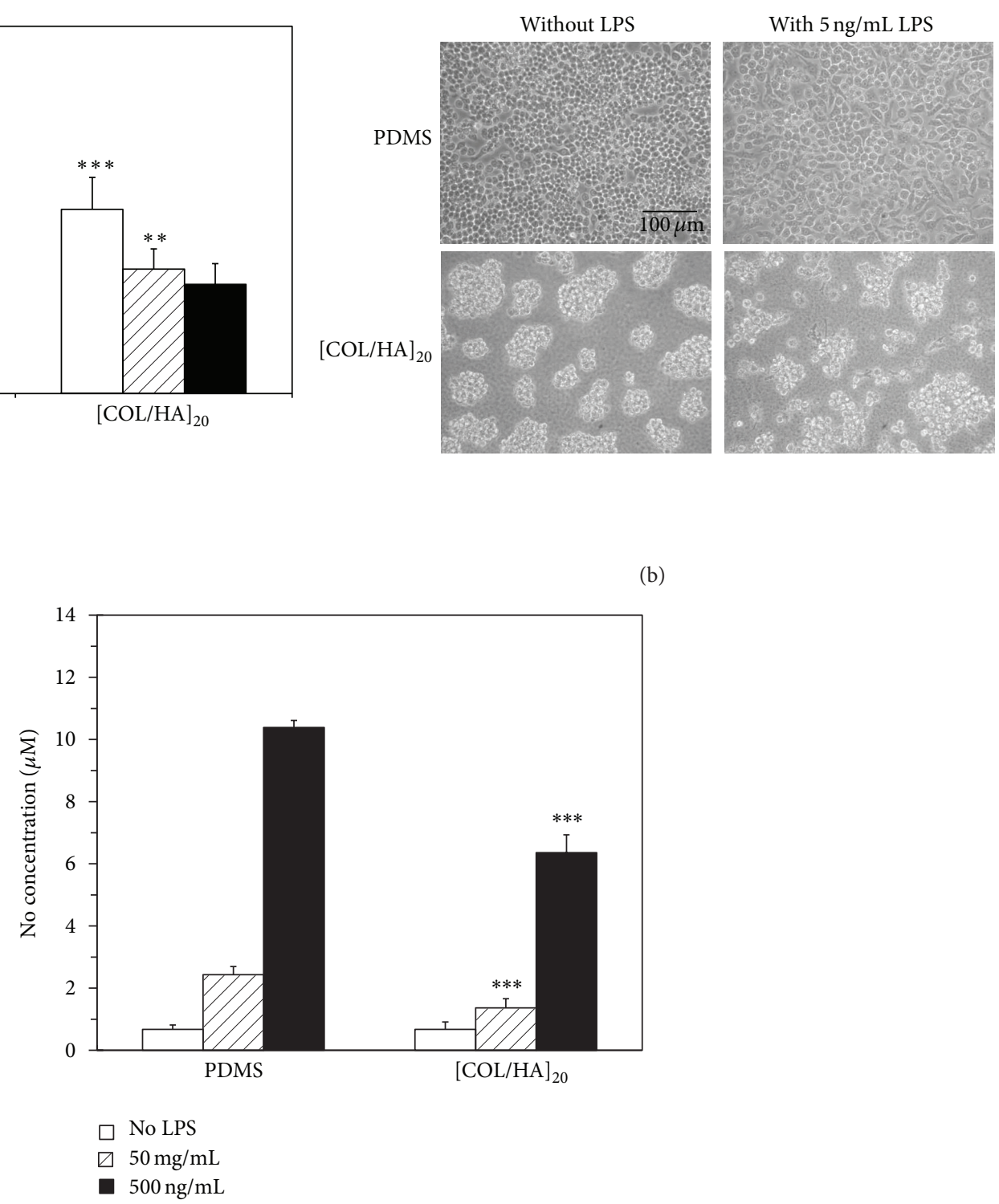

(b)

(c)

Figure 5: RAW 264.7 cells were seeded on PDMS or 20 bilayer-COL/HA films ([COL/HA $]_{20}$ ) for $12 \mathrm{~h}$, followed by $24 \mathrm{~h}$ incubation with and without LPS induction (50 or $500 \mathrm{ng} / \mathrm{mL}$ ). (a) The densities of RAW 264.7 cells; (b) microscopic images from the cells incubated without LPS or with $500 \mathrm{ng} / \mathrm{mL}$ LPS; (c) nitric oxide (NO) productions of RAW 264.7 cells. $n=6$ and value $=$ mean \pm SD. ${ }^{* * *} P<0.001$ versus PDMS at the same condition.

Morphologically, aggregations and fusion of macrophage-like cells were found on the border of the tissues surrounding the untreated PDMS (indicated by arrows), suggesting the formation of FBGCs (Figure 6(a)). On the other hand, few macrophage aggregates were found around the $[\mathrm{COL} / \mathrm{HA}]_{20}$ samples (Figure 6(a)). Therefore, a decrease in FBGC formation indicates less extent of an inflammatory response on $[\mathrm{COL} / \mathrm{HA}]_{20}$ in comparison to PDMS.

Further analysis of capsular thickness indicated the formation of thinner capsules surrounding the $[\mathrm{COL} / \mathrm{HA}]_{20}$ sample when comparing with the unmodified PDMS. The thickness of capsules around the epidermal and dermal sides of the $[\mathrm{COL} / \mathrm{HA}]_{20}$ samples was decreased by $29.14 \%$ and $56.61 \%$, respectively, compared to the same side of PDMS
(Figure 6(b)). The reduction in the capsule thickness by COL/HA multilayer films may be due to the downregulation of macrophage adhesion and activation.

This study demonstrates the potential of layer-by-layer $\mathrm{COL} / \mathrm{HA}$ multilayer films to reduce the foreign body reaction against implants. Both COL and HA are biodegradable and will be resorbed quickly after implantation. In this study, we only evaluated the outcome after 3-week implantation, and thus long-term efficacy remains under investigation. Nevertheless, the properties of PEM films can be further tuned by adjusting deposition parameters such as the types of polyelectrolytes, $\mathrm{pH}$, and ionic strength of polyelectrolyte solutions for finding better coatings. Furthermore, biomolecules such as anti-inflammatory drugs can be incorporated into PEM 

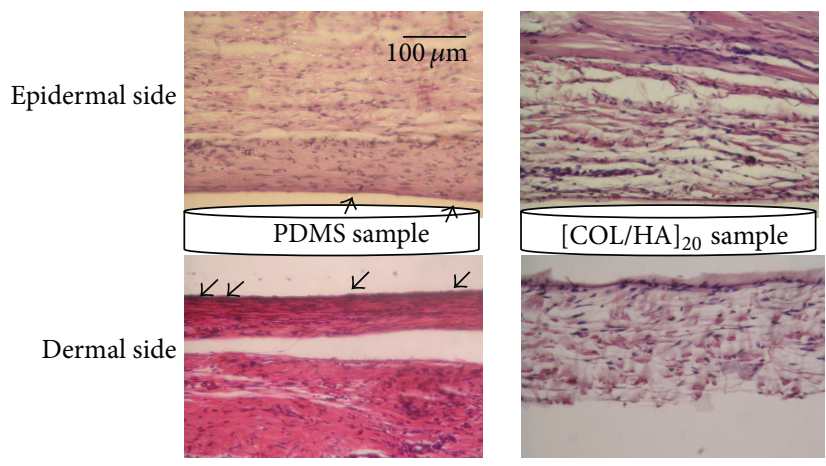

(a)

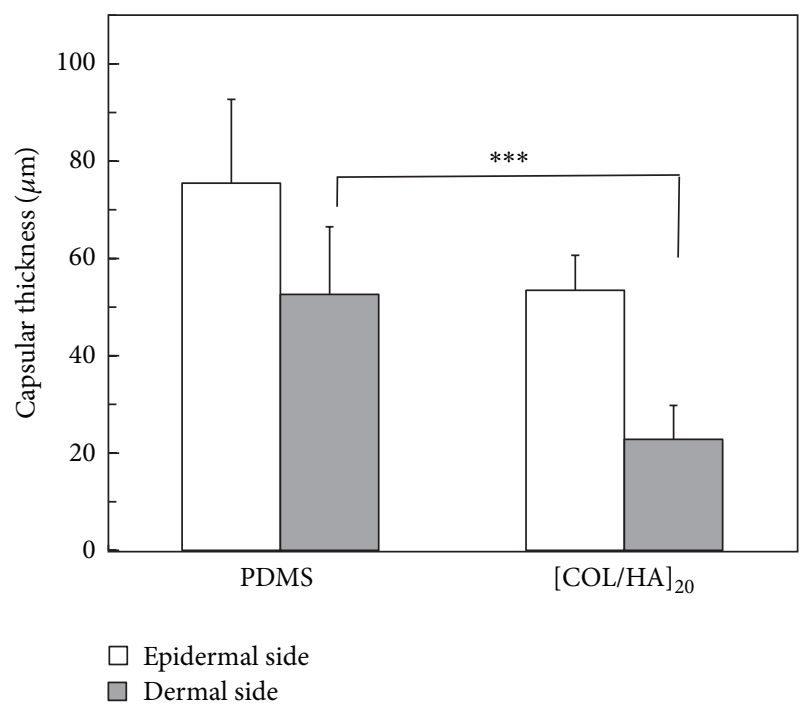

(b)

Figure 6: (a) Hematoxylin and Eosin staining of tissue sections around the PDMS and $[\mathrm{COL} / \mathrm{HA}]_{20}$-coated implants at the epidermal layer towards stratum cornea and the dermal layer towards hypodermis after 3 weeks of implantation. Arrows indicated FBGCs. (b) Statistical analysis of the capsular thickness around implants. $n=5$ or 6 and value $=$ mean \pm standard deviation.

films to prevent capsule formation [42, 43]. Currently, we look for a better formula of PEM films to prevent foreign body reaction to biomaterials.

\section{Conclusion}

In this study, we demonstrated that COL/HA multilayer films reduced the adhesion, proliferation, and activation of macrophages in vitro and capsule formation in vivo. Our results suggest the potential in fabricating a biocompatible material by reducing macrophage adhesion and activation to further reduce the capsule formation. Further investigation is required to find optimal PEM systems that minimize foreign body reaction or encapsulation for biomedical applications.

\section{Conflict of Interests}

The authors declare that there is no conflict of interests regarding the publication of this paper.

\section{Acknowledgment}

The authors gratefully acknowledge financial support from National Science Council, Taiwan (Grant nos. 96-2218-E002-009 and 97-3114-E-002-001).

\section{References}

[1] J. M. Anderson, A. Rodriguez, and D. T. Chang, "Foreign body reaction to biomaterials," Seminars in Immunology, vol. 20, no. 2, pp. 86-100, 2008.

[2] B. D. Ratner, "Reducing capsular thickness and enhancing angiogenesis around implant drug release systems," Journal of Controlled Release, vol. 78, no. 1-3, pp. 211-218, 2002.

[3] D. G. Wallace, J. Rosenblatt, and G. A. Ksander, "Tissue compatibility of collagen-silicone composites in a rat subcutaneous model," Journal of Biomedical Materials Research, vol. 26, no. 11, pp. 1517-1534, 1992.

[4] G. W. Hastings, "Definitions in biomaterials: progress in biomedical engineering 4, Editor: D.F. Williams. Elsevier, Amsterdam, 1987, pp viii + 72, US \$63.50," Biomaterials, vol. 10, no. 3, p. 216, 1989.

[5] Y. Ikada, "Surface modification of polymers for medical applications," Biomaterials, vol. 15, no. 10, pp. 725-736, 1994.

[6] B. D. Ratner and S. J. Bryant, "Biomaterials: where we have been and where we are going," Annual Review of Biomedical Engineering, vol. 6, pp. 41-75, 2004.

[7] J. M. Anderson and K. M. Miller, "Biomaterial biocompatibility and the macrophage," Biomaterials, vol. 5, no. 1, pp. 5-10, 1984.

[8] K. S. Jones, "Effects of biomaterial-induced inflammation on fibrosis and rejection," Seminars in Immunology, vol. 20, no. 2, pp. 130-136, 2008.

[9] J. Å. Johansson, T. Halthur, M. Herranen, L. Söderberg, U. Elofsson, and J. Hilborn, "Build-up of collagen and hyaluronic acid polyelectrolyte multilayers," Biomacromolecules, vol. 6, no. 3, pp. 1353-1359, 2005.

[10] D. Jiang, J. Liang, Y. Li, and P. W. Noble, "The role of Toll-like receptors in non-infectious lung injury," Cell Research, vol. 16, no. 8, pp. 693-701, 2006.

[11] D. Jiang, J. Liang, and P. W. Noble, "Hyaluronan in tissue injury and repair," Annual Review of Cell and Developmental Biology, vol. 23, pp. 435-461, 2007.

[12] B. T. Shannon, S. H. Love, and Q. N. Myrvik, "Participation of hyaluronic acid in the macrophage disappearance reaction," Immunological Communications, vol. 9, no. 4, pp. 357-370, 1980.

[13] M. K. Cho, S. H. Suh, C. H. Lee, and S. G. Kim, "Bovine type I collagen inhibits Raw264.7 cell proliferation through phosphoinositide 3-kinase- and mitogen-activated protein kinasedependent down-regulation of cyclins D1, A and B1," Biochimica et Biophysica Acta: Molecular Cell Research, vol. 1744, no. 1, pp. 47-57, 2005.

[14] G. A. Ksander and L. Gray, "Reduced capsule formation around soft silicone rubber prostheses coated with solid collagen," Annals of Plastic Surgery, vol. 14, no. 4, pp. 351-360, 1985.

[15] G. Decher, "Fuzzy nanoassemblies: toward layered polymeric multicomposites," Science, vol. 277, no. 5330, pp. 1232-1237, 1997.

[16] J. D. Mendelsohn, S. Y. Yang, J. Hiller, A. I. Hochbaum, and M. F. Rubner, "Rational design of cytophilic and cytophobic polyelectrolyte multilayer thin films," Biomacromolecules, vol. 4, no. 1, pp. 96-106, 2003. 
[17] H.-W. Chien, T.-Y. Chang, and W.-B. Tsai, "Spatial control of cellular adhesion using photo-crosslinked micropatterned polyelectrolyte multilayer films," Biomaterials, vol.30, no. 12, pp. 2209-2218, 2009.

[18] W.-B. Tsai, Y.-H. Chen, and H.-W. Chien, "Collaborative cellresistant properties of polyelectrolyte multilayer films and surface PEGylation on reducing cell adhesion to cytophilic surfaces," Journal of Biomaterials Science, Polymer Edition, vol. 20, no. 11, pp. 1611-1628, 2009.

[19] W.-B. Tsai, R. P.-Y. Chen, K.-L. Wei et al., "Polyelectrolyte multilayer films functionalized with peptides for promoting osteoblast functions," Acta Biomaterialia, vol. 5, no. 9, pp. 34673477, 2009.

[20] H.-W. Chien, S.-P. Wu, W.-H. Kuo et al., "Modulation of hemocompatibility of polysulfone by polyelectrolyte multilayer films," Colloids and Surfaces B: Biointerfaces, vol. 77, no. 2, pp. 270-278, 2010.

[21] C. Picart, "Polyelectrolyte multilayer film: from physicochemical properties to the control of cellular processes," Current Medicinal Chemistry, vol. 15, no. 7, pp. 685-697, 2008.

[22] J. Zhang, B. Senger, D. Vautier et al., "Natural polyelectrolyte films based on layer-by layer deposition of collagen and hyaluronic acid," Biomaterials, vol. 26, no. 16, pp. 3353-3361, 2005.

[23] W.-B. Tsai and J.-H. Lin, "Modulation of morphology and functions of human hepatoblastoma cells by nano-grooved substrata," Acta Biomaterialia, vol. 5, no. 5, pp. 1442-1454, 2009.

[24] W.-B. Tsai, Q. Shi, J. M. Grunkemeier, C. McFarland, and T. A. Horbett, "Platelet adhesion to radiofrequency glow-dischargedeposited fluorocarbon polymers preadsorbed with selectively depleted plasmas show the primary role of fibrinogen," Journal of Biomaterials Science, Polymer Edition, vol. 15, no. 7, pp. 817840, 2004.

[25] Y. Lu, J. Sun, and J. Shen, "Cell adhesion properties of patterned poly(acrylic acid)/poly(allylamine hydrochloride) multilayer films created by room-temperature imprinting technique," Langmuir, vol. 24, no. 15, pp. 8050-8055, 2008.

[26] H.-W. Chien, S.-F. Tan, K.-L. Wei, and W.-B. Tsai, "Modulation of the functions of osteoblast-like cells on poly(allylamine hydrochloride) and poly(acrylic acid) multilayer films," Colloids and Surfaces B: Biointerfaces, vol. 88, no. 1, pp. 297-303, 2011.

[27] D. L. Elbert, C. B. Herbert, and J. A. Hubbell, “Thin polymer layers formed by polyelectrolyte multilayer techniques on biological surfaces," Langmuir, vol. 15, no. 16, pp. 5355-5362, 1999.

[28] S. Mehrotra, S. C. Hunley, K. M. Pawelec et al., "Cell adhesive behavior on thin polyelectrolyte multilayers: cells attempt to achieve homeostasis of its adhesion energy," Langmuir, vol. 26, no. 15, pp. 12794-12802, 2010.

[29] D. E. Discher, P. Janmey, and Y.-L. Wang, "Tissue cells feel and respond to the stiffness of their substrate," Science, vol. 310, no. 5751, pp. 1139-1143, 2005.

[30] L. Richert, A. J. Engler, D. E. Discher, and C. Picart, "Elasticity of native and cross-linked polyelectrolyte multilayer films," Biomacromolecules, vol. 5, no. 5, pp. 1908-1916, 2004.

[31] R. K. Saxena, V. Vallyathan, and D. M. Lewis, "Evidence for lipopolysaccharide-induced differentiation of RAW264.7 murine macrophage cell line into dendritic like cells," Journal of Biosciences, vol. 28, no. 1, pp. 129-134, 2003.

[32] K. M. Sheehan, L. B. Delott, R. A. West, J. D. Bonnema, and D. H. Deheer, "Hyaluronic acid of high molecular weight inhibits proliferation and induces cell death in U937 macrophage cells," Life Sciences, vol. 75, no. 26, pp. 3087-3102, 2004.
[33] C.-Y. Chang, M. Tucci, and R. C. Baker, "Lipopolysaccharidestimulated nitric oxide production and inhibition of cell proliferation is antagonized by ethanol in a clonal macrophage cell line," Alcohol, vol. 20, no. 1, pp. 37-43, 2000.

[34] M. C. Berg, S. Y. Yang, P. T. Hammond, and M. F. Rubner, "Controlling mammalian cell interactions on patterned polyelectrolyte multilayer surfaces," Langmuir, vol. 20, no. 4, pp. 1362-1368, 2004.

[35] T. Boudou, T. Crouzier, K. Ren, G. Blin, and C. Picart, "Multiple functionalities of polyelectrolyte multilayer films: new biomedical applications," Advanced Materials, vol. 22, no. 4, pp. 441-467, 2010.

[36] Y. Suzuki and T. Yamaguchi, "Effects of hyaluronic acid on macrophage phagocytosis and active oxygen release," Agents and Actions, vol. 38, no. 1-2, pp. 32-37, 1993.

[37] M. K. Cho, S. H. Suh, and S. G. Kim, "JunB/AP-1 and NF$\kappa \mathrm{B}$-mediated induction of nitric oxide synthase by bovine type I collagen in serum-stimulated murine macrophages," Nitric Oxide: Biology and Chemistry, vol. 6, no. 3, pp. 319-332, 2002.

[38] M. K. Cho, Y. H. Cho, G. H. Lee, and S. G. Kim, "Induction of cyclooxygenase- 2 by bovine type I collagen in macrophages via $\mathrm{C} / \mathrm{EBP}$ and $\mathrm{CREB}$ activation by multiple cell signaling pathways," Biochemical Pharmacology, vol. 67, no. 12, pp. 22392250, 2004.

[39] D. T. Luttikhuizen, M. C. Harmsen, and M. J. A. Van Luyn, "Cellular and molecular dynamics in the foreign body reaction," Tissue Engineering, vol. 12, no. 7, pp. 1955-1970, 2006.

[40] P. Tripathi, P. Tripathi, L. Kashyap, and V. Singh, "The role of nitric oxide in inflammatory reactions," FEMS Immunology and Medical Microbiology, vol. 51, no. 3, pp. 443-452, 2007.

[41] D. Saraydin, S. Ünver-Saraydin, E. Karadağ, E. Koptagel, and O. Güven, "In vivo biocompatibility of radiation crosslinked acrylamide copolymers," Nuclear Instruments and Methods in Physics Research B: Beam Interactions with Materials and Atoms, vol. 217, no. 2, pp. 281-292, 2004.

[42] M. Helmuth and V. Dmitry, Drug Delivery: Polyelectrolyte Multilayers, Encyclopedia of Surface and Colloid Science, Taylor \& Francis, 2nd edition, 2007.

[43] V. Krzyzanek, N. Sporenberg, U. Keller, J. Guddorf, R. Reichelt, and M. Schönhoff, "Polyelectrolyte multilayer capsules: nanostructure and visualisation of nanopores in the wall," Soft Matter, vol. 7, no. 15, pp. 7034-7041, 2011. 

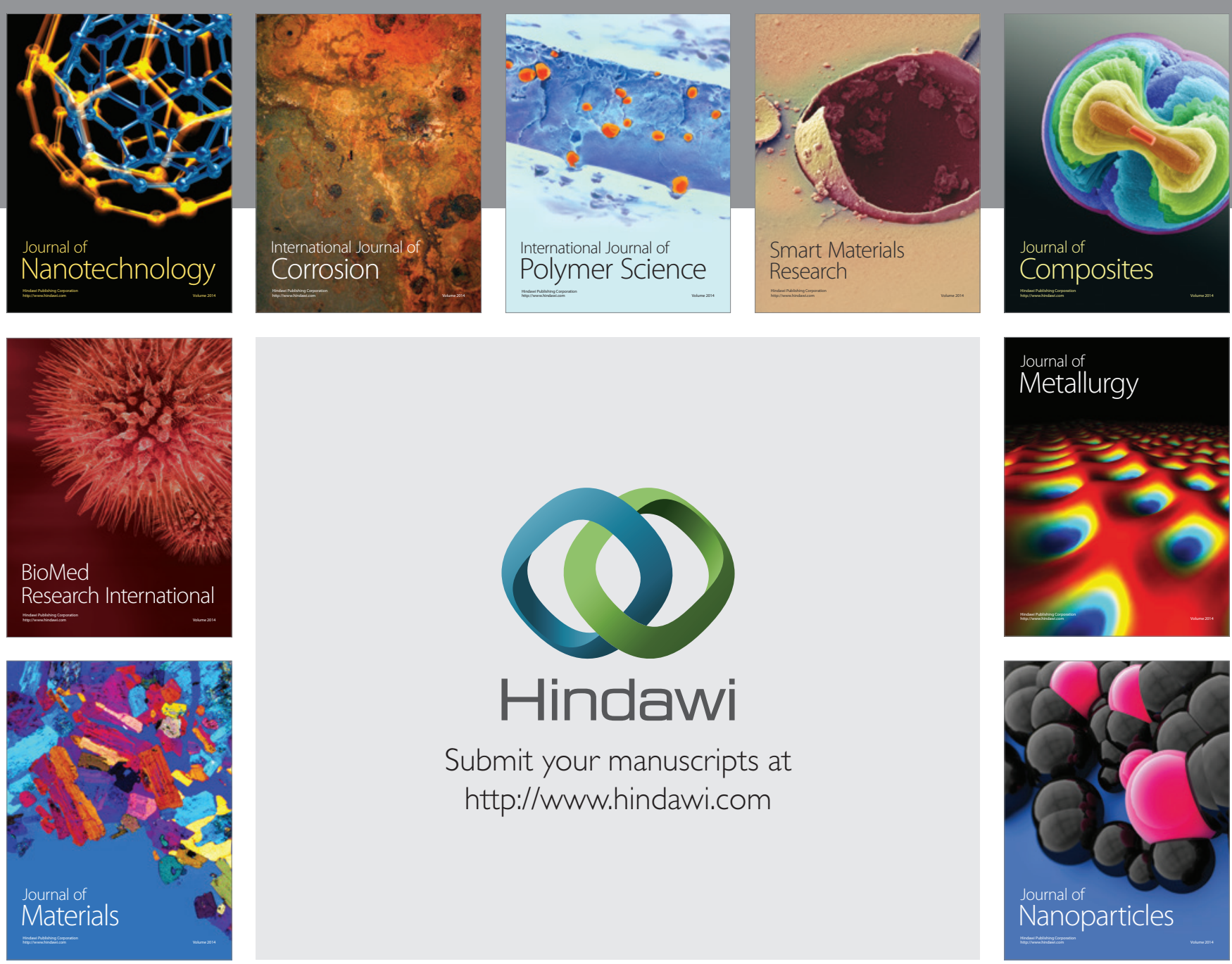

Submit your manuscripts at http://www.hindawi.com
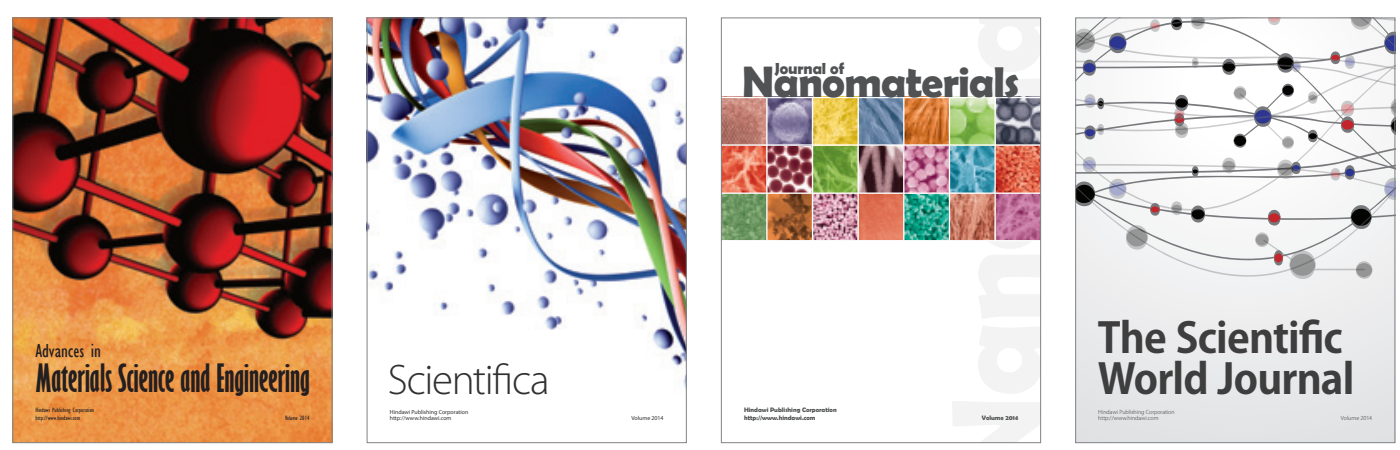

\section{The Scientific World Journal}
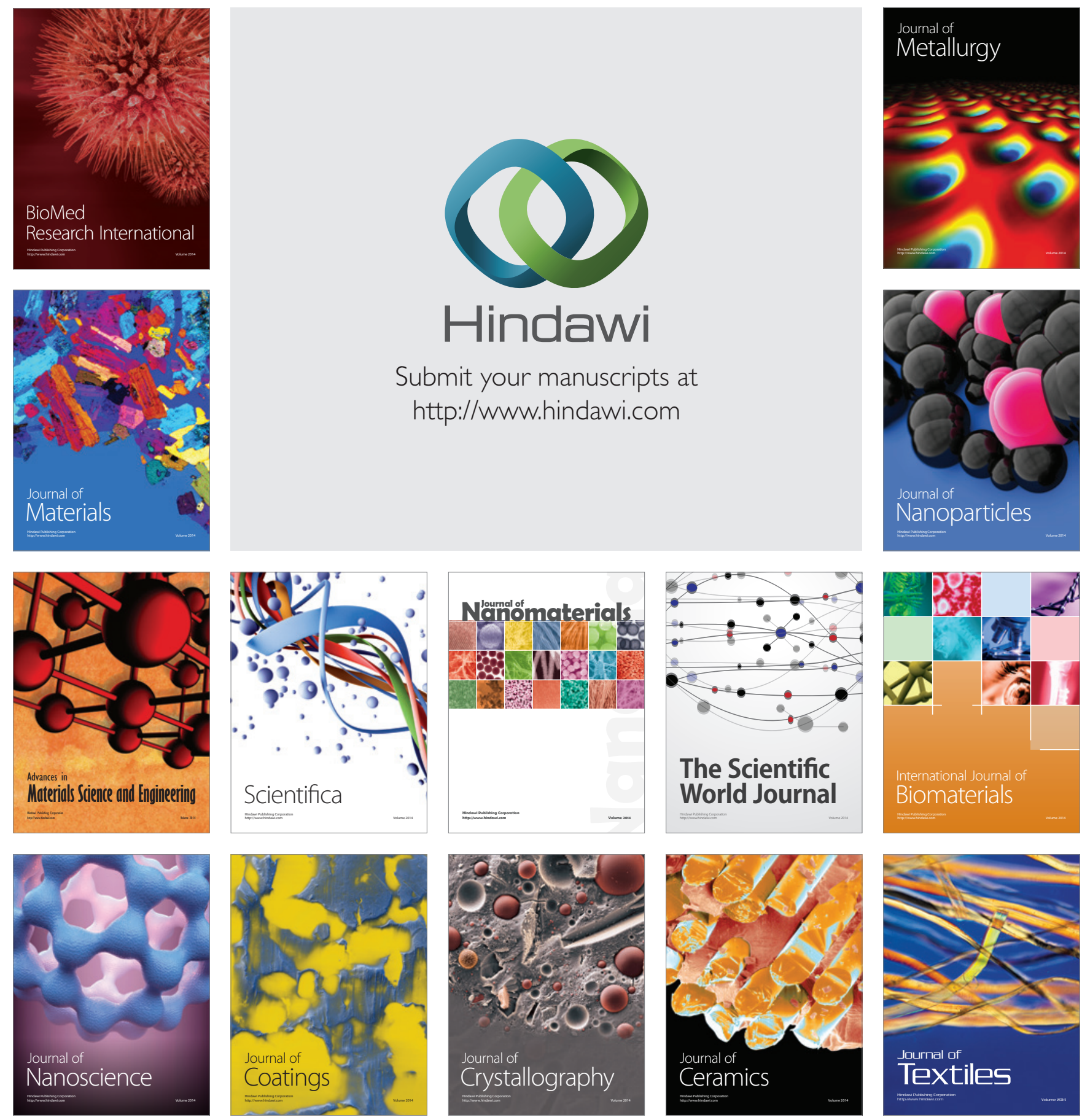